\title{
Revealing the Doping Mechanism of spiro-MeOTAD via Zn Complexation in the
}

\section{Absence of Oxygen and Light}

Yasemin Saygili, ${ }^{\mathrm{a} \dagger}$ Hui-Seon Kim,,${ }^{\mathrm{a}, \mathrm{b} \dagger^{*}}$ Bowen Yang, ${ }^{\mathrm{a}} \mathrm{Jiajia}$ Suo, ${ }^{\mathrm{a}}$ Ana B. Muñoz-Garcia, ${ }^{\mathrm{c}}$ Michele Pavone, ${ }^{\mathrm{d}}$ and Anders Hagfeld $\mathrm{t}^{\mathrm{a}^{*}}$

aLaboratory of Photomolecular Science, Institute of Chemical Sciences and Engineering, School of Basic Sciences, Ecole Polytechnique Fédérale de Lausanne, CH-1015 Lausanne, Switzerland.

${ }^{b}$ Department of Chemistry, Inha Uiversity, Incheon 22212, Korea.

'Department of Physics "Ettore Pancini", Università degli studi di Napoli Federico II, Complesso Universitario Monte Sant'Angelo via Cintia 21, 80126 Naples, Italy.

${ }^{\mathrm{d}}$ Department of Chemical Sciences, Università degli studi di Napoli Federico II, Complesso Universitario Monte Sant'Angelo via Cintia 21, 80126 Naples, Italy.

${ }^{\dagger}$ Both authors contributed equally to this work.

* Corresponding authors

H.-S.K.: hui-seon.kim@inha.ac.kr and A. H.: anders.hagfeldt@epfl.ch 


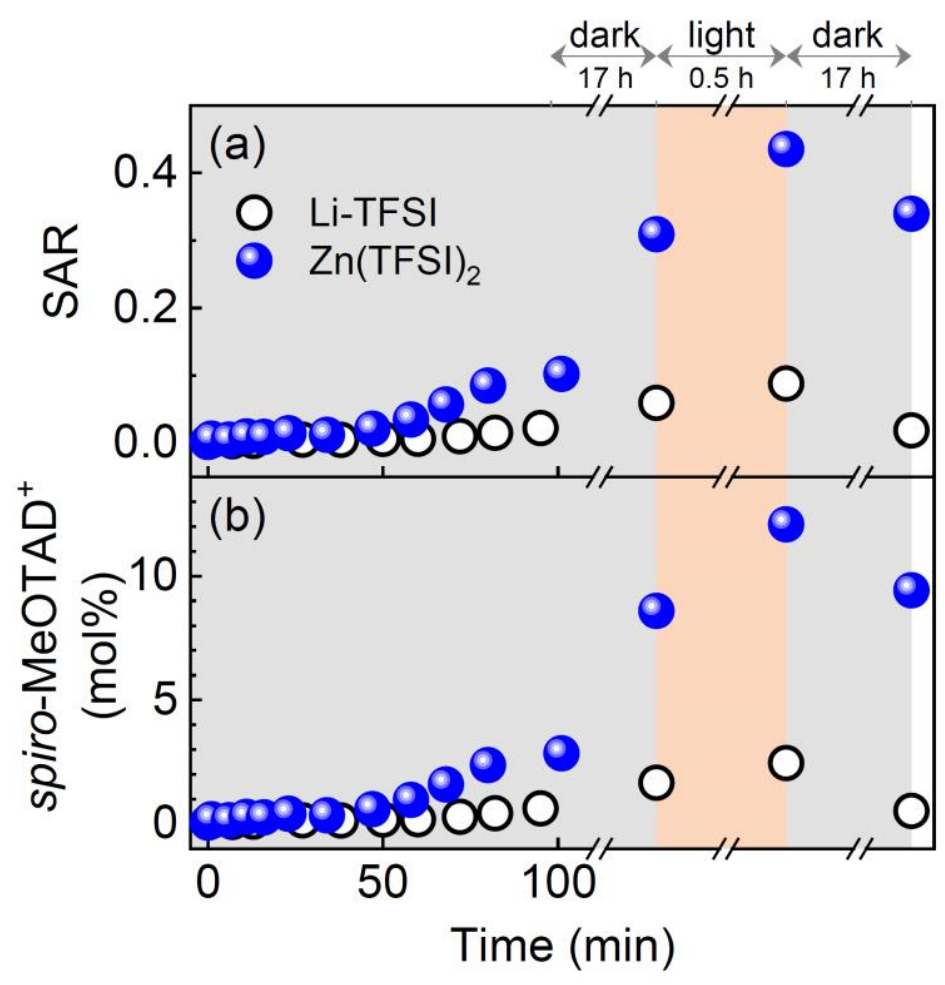

Figure S1 (a) Standardized absorptance ratio (SAR) and (b) $\mathrm{mol}^{\%}$ spiro-MeOTAD ${ }^{+}$as a function of time. The spiro-MeOTAD solution was doped either by LiTFSI (black open circles) or $\mathrm{Zn}$ (TFSI) 2 (blue closed circles). The SAR was calculated by absorbance ratio at $523 \mathrm{~nm}$ and $407 \mathrm{~nm}\left(\mathrm{SAR}=\mathrm{A}_{523 \mathrm{~nm}} / \mathrm{A}_{407 \mathrm{~nm}}\right)$. The mol\% spiro-MeOTAD ${ }^{+}$was subsequently determined from the calibration curve of spiro-MeOTAD through SAR values. ${ }^{45}$
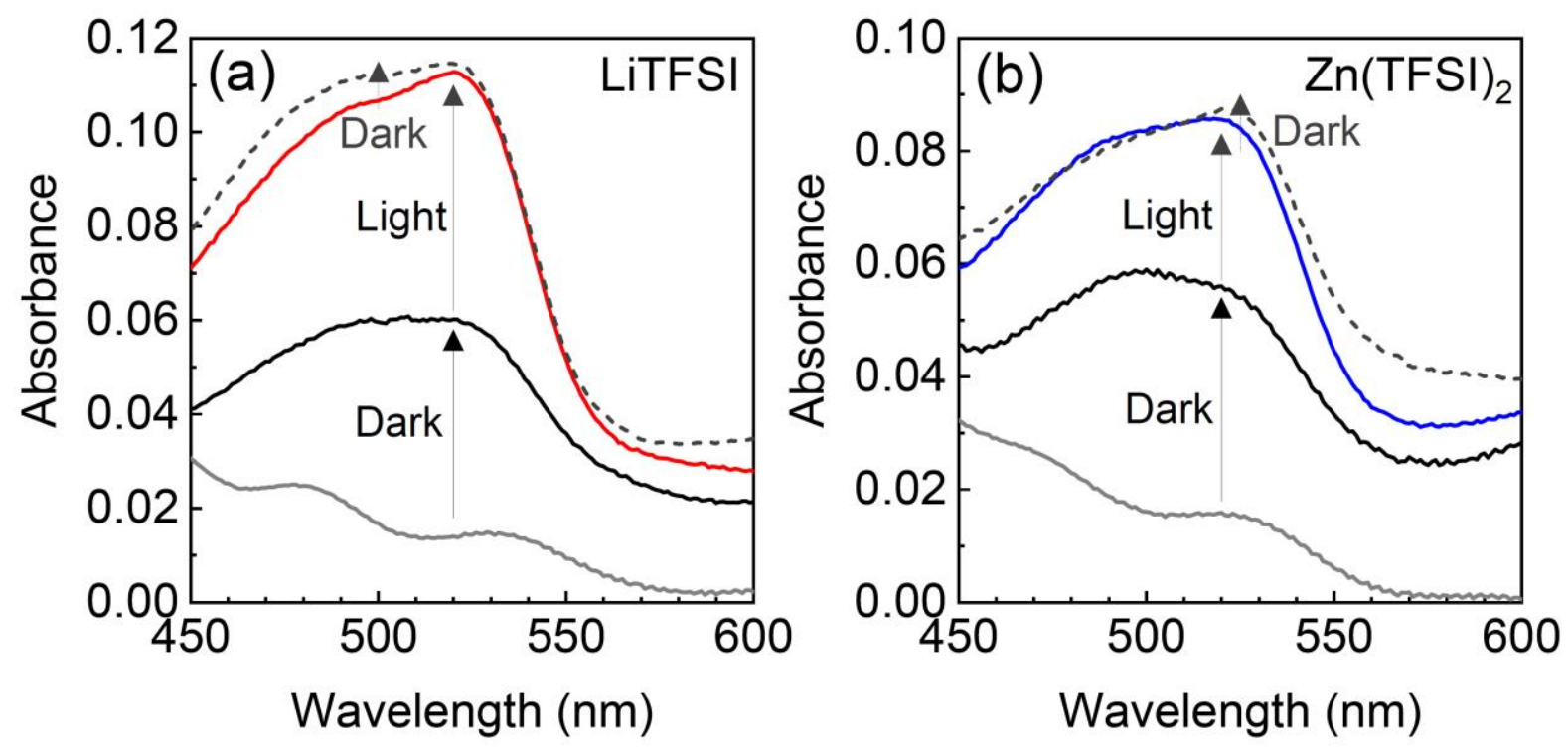

Figure S2 Absorbance spectra of spiro-MeOTAD films doped by (a) LiTFSI or (b)Zn(TFSI) 2 . The spiro-MeOTAD layer was deposited on a glass substrate and measured as prepared (grey 
solid), after being saturated in dark (black), after light soaking for $30 \mathrm{~min}$ (red for LiTFSI and blue for $\mathrm{Zn}(\mathrm{TFSI})_{2}$ ) and after being kept again in dark overnight (grey dash).
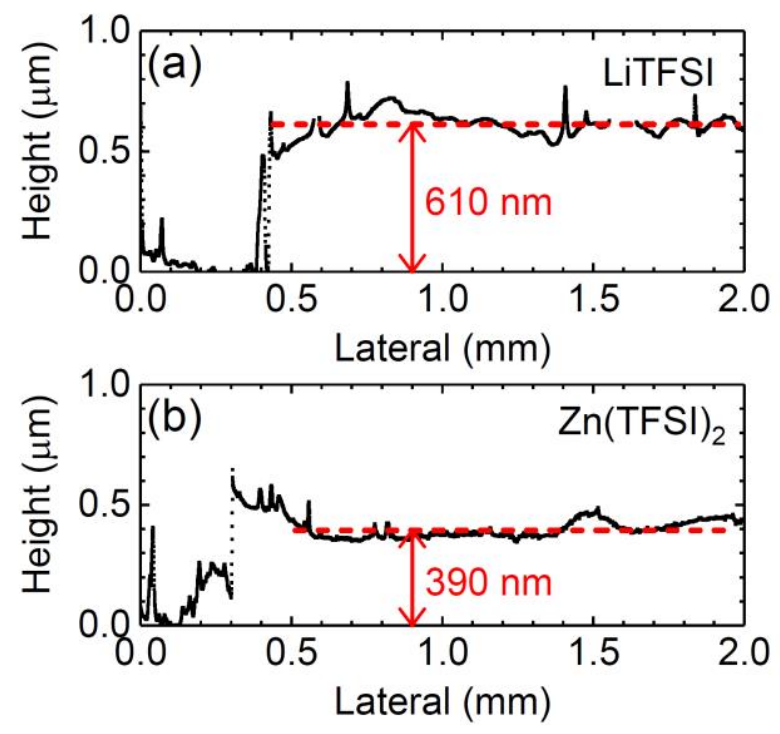

Figure S3 Thickness of spiro-MeOTAD film doped by (a) LiTFSI or (b)Zn(TFSI)2.

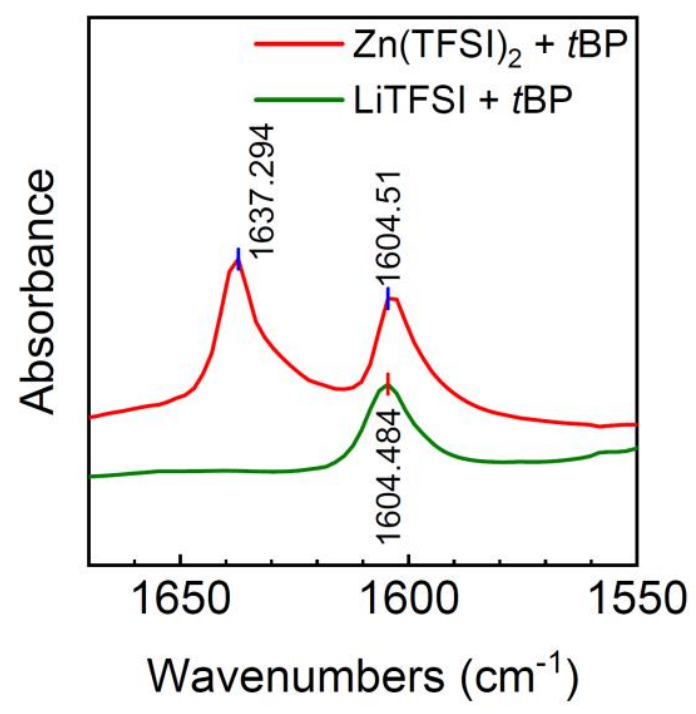

Figure S4 FT-IR spectra of mixture of Zn(TFSI) $)_{2}$ and tBP (red) and LiTFSI and $t$ BP (green). 
(a)
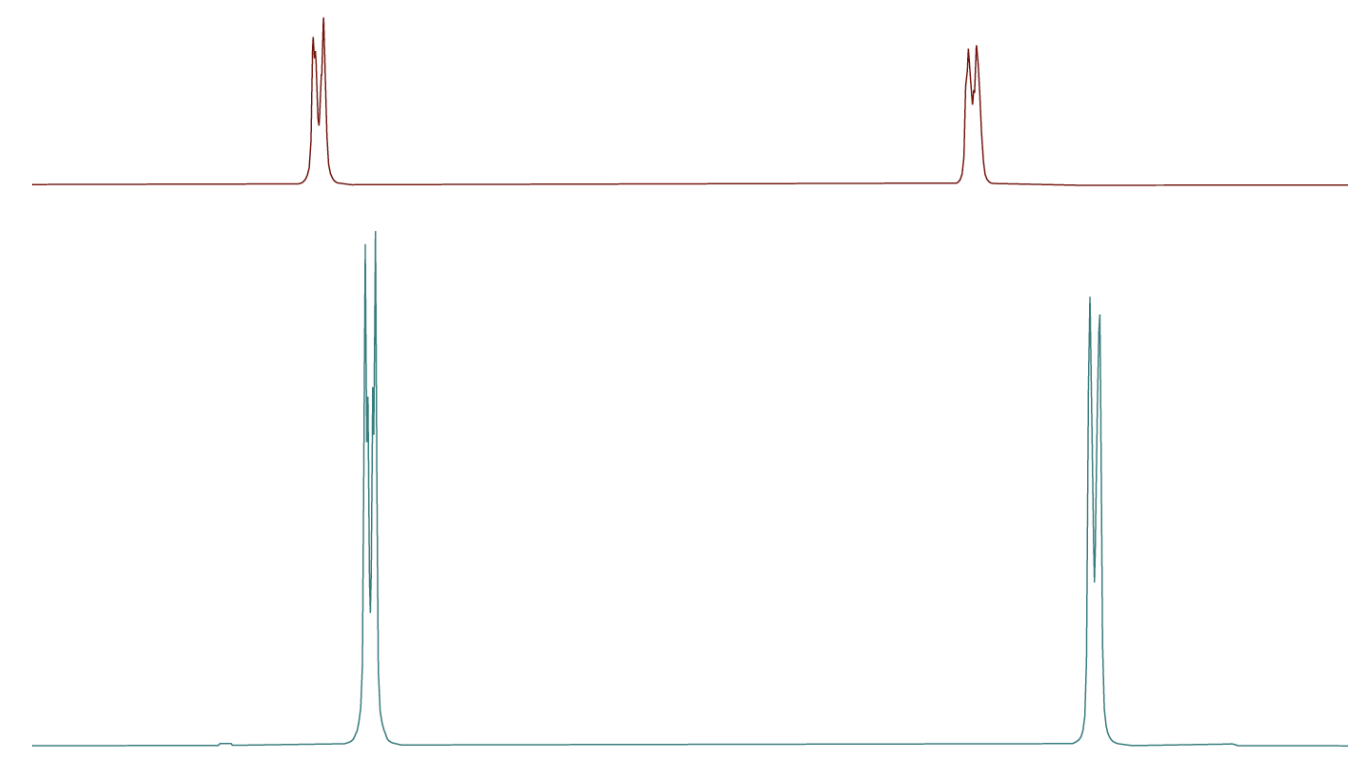

\section{\begin{tabular}{llllllllll}
\hline 8.9 & 8.7 & 8.5 & 8.3 & 8.1 & 7.9 & 7.7 & 7.5 & 7.3 & 7.1
\end{tabular}}

(b)

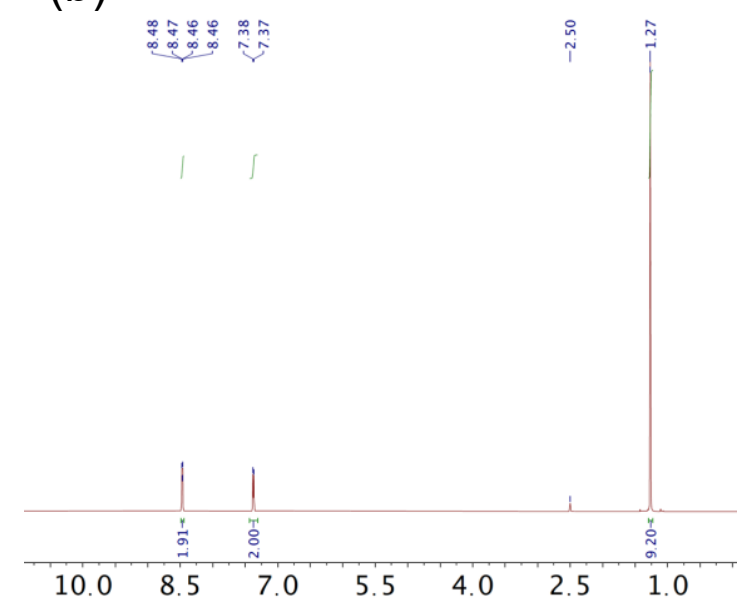

(c)

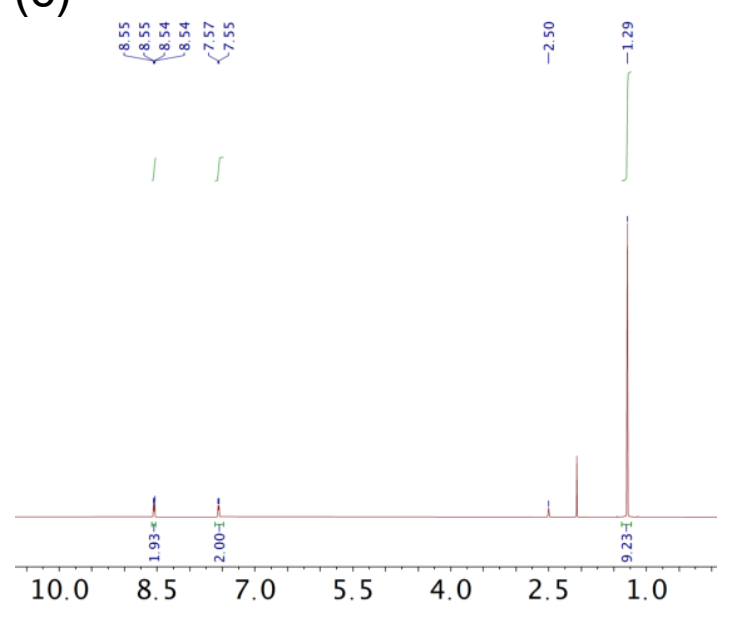

Figure S5 (a) Comparison of 1H NMR spectra of $(t \mathrm{BP})_{\mathrm{m}} \cdots \mathrm{Zn}(\mathrm{TFSI})_{2}$ complex (top) and $t \mathrm{BP}$ (bottom) in DMSO-d $\mathrm{d}_{6}$. (b) $1 \mathrm{H}$ NMR raw spectrum of $t \mathrm{BP}$ in DMSO-d $\mathrm{d}_{6}$. (c) $1 \mathrm{H}$ NMR raw spectrum of $(t \mathrm{BP})_{\mathrm{m}} \cdots \cdot \mathrm{Zn}(\mathrm{TFSI})_{2}$ complex in DMSO-d $\mathrm{d}_{6}$.

$$
\begin{aligned}
& \text { spiro-MeOTAD }+\mathrm{Li}^{+}\left(\mathrm{TFSI}^{-}\right)+\mathrm{m} \cdot t \mathrm{BP} \\
& \rightarrow \text { spiro-MeOTAD } \\
& +\left[\mathrm{Li}(t \mathrm{BP})_{\mathrm{m}}\right]\left(\mathrm{TFSI}^{-}\right)
\end{aligned}
$$

Scheme S1. Mechanism of spiro-MeOTAD oxidation by Li-tBP-TFSI complex formation. 
Table S1 Computed reaction energies $(\Delta \mathrm{E}$ in $\mathrm{eV})$ corresponding to the reaction in Scheme $\mathrm{S} 1$. $\mathrm{m}$ refers to the numbers of $t \mathrm{BP}$ coordinating the $\mathrm{Li}^{+}$cation.

\begin{tabular}{cr}
\hline$t \mathrm{BP}$ & $\Delta \mathrm{E}(\mathrm{eV})$ \\
\hline $\mathrm{m}=0$ & 4.38 \\
$\mathrm{~m}=1$ & 3.41 \\
$\mathrm{~m}=2$ & 2.24 \\
$\mathrm{~m}=3$ & $*$ \\
$\mathrm{~m}=4$ & 4.38
\end{tabular}

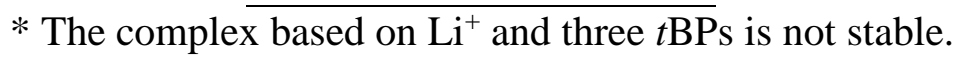
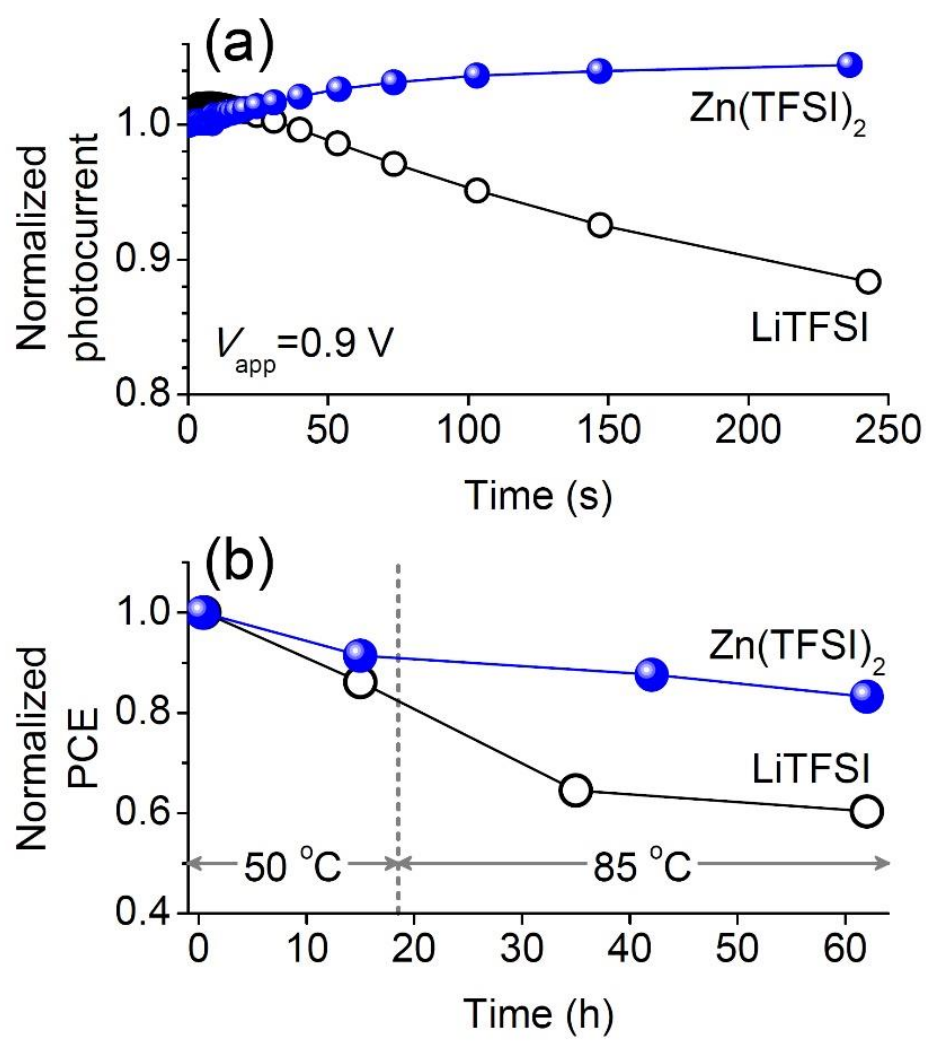

Figure S6 (a) Normalized photocurrent of PSCs based on LiTFSI (black open symbols) and $\mathrm{Zn}$ (TFSI) 2 (blue closed symbols) as a dopant for spiro-MeOTAD. The device was kept near the maximum power point $(\sim 0.9 \mathrm{~V})$ under full sun intensity. (b) Thermal stability of PSCs based on LiTFSI (black open symbols) and Zn(TFSI) 2 (blue closed symbols) as a dopant for spiro-MeOTAD. The devices were kept in dark while being heated on a hot plate at $\sim 50 \%$ R.H. 
(a) spiro-MeOTAD

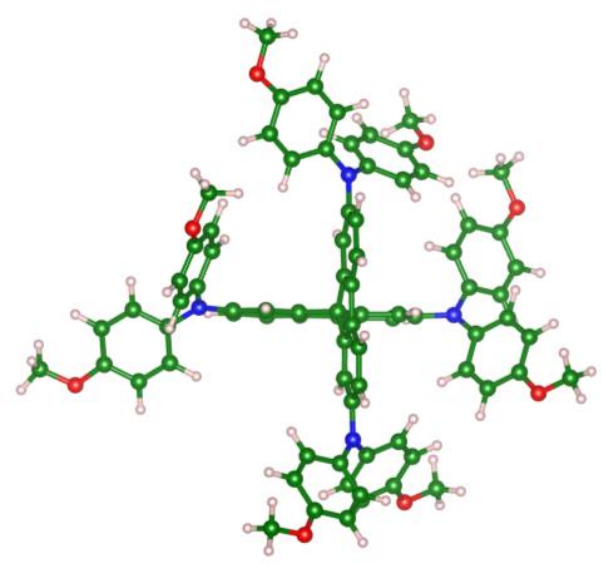

$\begin{array}{lrrr}\mathrm{O} & 17.917345 & 15.858156 & 6.531467 \\ \mathrm{O} & 10.692282 & -0.174876 & 12.364471 \\ \mathrm{O} & 16.178513 & 13.844006 & 2.774226 \\ \mathrm{~N} & 12.579628 & 9.575483 & 2.297650 \\ \mathrm{C} & 12.180120 & 6.322287 & 6.913579 \\ \mathrm{~N} & 16.082049 & 10.971968 & 8.561244 \\ \mathrm{O} & 10.390945 & 2.819644 & 0.817767 \\ \mathrm{O} & 12.389294 & 10.787772 & 12.770115 \\ \mathrm{~N} & 13.450907 & 4.272868 & 10.375855 \\ \mathrm{~N} & 15.463977 & 2.908937 & 3.186313 \\ \mathrm{C} & 10.931473 & 7.556687 & 4.970030 \\ \mathrm{C} & 13.550213 & 6.179222 & 7.212440 \\ \mathrm{C} & 13.336963 & 7.487002 & 5.254702 \\ \mathrm{C} & 15.197375 & 8.971095 & 7.451489 \\ \mathrm{C} & 15.430771 & 7.847544 & 6.679096 \\ \mathrm{C} & 15.290419 & 5.887721 & 5.362801 \\ \mathrm{C} & 16.283693 & 9.789474 & 7.803060 \\ \mathrm{C} & 13.985027 & 5.492443 & 8.330061 \\ \mathrm{C} & 14.890575 & 4.773024 & 4.651565 \\ \mathrm{C} & 14.404211 & 6.856720 & 6.146208 \\ \mathrm{O} & 17.992982 & 5.977805 & 13.172404 \\ \mathrm{C} & 16.725358 & 7.508595 & 6.235787 \\ \mathrm{C} & 17.509951 & 14.407043 & 8.338155 \\ \mathrm{C} & 16.950996 & 13.650644 & 6.105645 \\ \mathrm{C} & 11.233175 & 5.750237 & 7.767123 \\ \mathrm{C} & 16.638333 & 6.290543 & 5.424071 \\ \mathrm{C} & 12.406637 & 8.735874 & 3.429267 \\ \mathrm{C} & 17.456197 & 14.639759 & 3.515325 \\ \mathrm{C} & 13.517124 & & \end{array}$




\begin{tabular}{|c|c|c|c|}
\hline $\mathrm{C}$ & 17.805989 & 8.323318 & 6.587533 \\
\hline $\mathrm{C}$ & 15.857081 & 4.028706 & 3.948545 \\
\hline $\mathrm{C}$ & 16.545224 & 12.202966 & 8.020995 \\
\hline $\mathrm{C}$ & 13.500233 & 10.650372 & 2.377407 \\
\hline $\mathrm{C}$ & 17.579554 & 9.453908 & 7.372243 \\
\hline $\mathrm{C}$ & 17.208671 & 4.425313 & 4.003715 \\
\hline $\mathrm{C}$ & 12.047723 & 7.124531 & 5.693031 \\
\hline $\mathrm{C}$ & 12.746433 & 3.136657 & 10.851852 \\
\hline $\mathrm{C}$ & 15.327060 & 12.782464 & 2.581758 \\
\hline $\mathrm{C}$ & 11.659897 & 5.071964 & 8.908595 \\
\hline $\mathrm{C}$ & 17.601989 & 5.541425 & 4.742857 \\
\hline $\mathrm{C}$ & 12.458035 & 2.998331 & 12.219941 \\
\hline $\mathrm{C}$ & 13.529745 & 8.279097 & 4.138001 \\
\hline $\mathrm{C}$ & 11.965575 & 9.234351 & 1.069817 \\
\hline $\mathrm{C}$ & 13.031198 & 4.939292 & 9.205797 \\
\hline $\mathrm{C}$ & 11.485554 & 10.224435 & 0.205304 \\
\hline $\mathrm{C}$ & 12.077670 & 1.797859 & 2.094631 \\
\hline $\mathrm{C}$ & 16.489118 & 12.446961 & 6.646055 \\
\hline $\mathrm{C}$ & 11.647552 & 2.906655 & 1.351205 \\
\hline $\mathrm{C}$ & 11.219987 & 7.550580 & -0.513383 \\
\hline $\mathrm{C}$ & 14.190138 & 2.900698 & 2.554490 \\
\hline $\mathrm{C}$ & 11.116718 & 8.363791 & 3.846074 \\
\hline $\mathrm{C}$ & 14.421623 & 12.524486 & 3.620345 \\
\hline $\mathrm{C}$ & 11.825628 & 7.887563 & 0.689992 \\
\hline $\mathrm{C}$ & 14.592184 & 4.732089 & 11.086317 \\
\hline $\mathrm{C}$ & 15.316228 & 11.973699 & 1.441219 \\
\hline $\mathrm{C}$ & 12.499131 & 4.007341 & 1.200893 \\
\hline $\mathrm{C}$ & 14.788677 & 6.096349 & 11.321491 \\
\hline $\mathrm{C}$ & 15.545869 & 3.820419 & 11.568882 \\
\hline $\mathrm{C}$ & 11.783033 & 1.880401 & 12.691981 \\
\hline $\mathrm{C}$ & 10.751663 & 8.547851 & -1.379288 \\
\hline $\mathrm{C}$ & 15.131944 & 10.967903 & 9.611829 \\
\hline $\mathrm{C}$ & 11.633737 & 1.012610 & 10.437711 \\
\hline $\mathrm{C}$ & 12.334659 & 2.129413 & 9.974278 \\
\hline $\mathrm{C}$ & 14.230432 & 12.020670 & 9.796791 \\
\hline $\mathrm{C}$ & 16.859411 & 5.634528 & 12.487217 \\
\hline $\mathrm{C}$ & 13.257618 & 10.911084 & 11.717537 \\
\hline $\mathrm{C}$ & 11.359862 & 0.880951 & 11.803891 \\
\hline $\mathrm{C}$ & 14.412743 & 10.910264 & 1.350194 \\
\hline $\mathrm{C}$ & 17.069432 & 13.198629 & 8.862258 \\
\hline $\mathrm{C}$ & 10.892929 & 9.891774 & -1.015854 \\
\hline $\mathrm{C}$ & 14.150247 & 9.845016 & 11.531026 \\
\hline $\mathrm{C}$ & 13.764890 & 3.992336 & 1.792299 \\
\hline $\mathrm{C}$ & 16.660267 & 4.263830 & 12.269499 \\
\hline
\end{tabular}




\begin{tabular}{|c|c|c|c|}
\hline $\mathrm{C}$ & 15.918310 & 6.551262 & 12.005725 \\
\hline $\mathrm{C}$ & 13.306022 & 12.003574 & 10.847281 \\
\hline $\mathrm{C}$ & 13.329412 & 1.800308 & 2.696413 \\
\hline $\mathrm{C}$ & 15.079335 & 9.878167 & 10.501388 \\
\hline $\mathrm{C}$ & 17.134684 & 14.121912 & 1.761408 \\
\hline $\mathrm{C}$ & 18.242288 & 7.358990 & 13.395759 \\
\hline $\mathrm{C}$ & 9.899451 & 3.933598 & 0.084916 \\
\hline $\mathrm{C}$ & 10.253506 & -1.217804 & 11.506591 \\
\hline $\mathrm{C}$ & 11.450219 & 11.830747 & 12.982408 \\
\hline $\mathrm{C}$ & 16.291075 & 1.760582 & 3.093812 \\
\hline $\mathrm{C}$ & 16.434828 & 1.085669 & 1.878229 \\
\hline $\mathrm{C}$ & 16.964260 & 1.263493 & 4.223519 \\
\hline $\mathrm{C}$ & 17.223078 & -0.064841 & 1.780633 \\
\hline $\mathrm{H}$ & 15.916504 & 1.456442 & 1.000339 \\
\hline $\mathrm{C}$ & 17.767446 & 0.135061 & 4.129570 \\
\hline $\mathrm{H}$ & 16.856344 & 1.772954 & 5.174973 \\
\hline $\mathrm{C}$ & 17.900682 & -0.541378 & 2.908048 \\
\hline $\mathrm{H}$ & 17.306180 & -0.564178 & 0.823200 \\
\hline $\mathrm{H}$ & 18.290058 & -0.250544 & 4.998553 \\
\hline $\mathrm{O}$ & 18.705743 & -1.649055 & 2.921749 \\
\hline $\mathrm{C}$ & 18.874439 & -2.365549 & 1.707731 \\
\hline $\mathrm{H}$ & 19.331757 & -1.737054 & 0.932847 \\
\hline $\mathrm{H}$ & 19.541378 & -3.196307 & 1.940675 \\
\hline $\mathrm{H}$ & 17.918056 & -2.758047 & 1.338953 \\
\hline $\mathrm{C}$ & 17.883602 & 16.125043 & 5.136395 \\
\hline $\mathrm{H}$ & 18.275735 & 17.135635 & 5.015871 \\
\hline $\mathrm{H}$ & 18.515203 & 15.417683 & 4.583365 \\
\hline $\mathrm{H}$ & 16.861687 & 16.074549 & 4.741597 \\
\hline $\mathrm{O}$ & 10.178450 & 8.109858 & -2.544288 \\
\hline $\mathrm{C}$ & 9.686769 & 9.084992 & -3.451821 \\
\hline $\mathrm{H}$ & 10.489948 & 9.746693 & -3.800733 \\
\hline $\mathrm{H}$ & 9.281810 & 8.529388 & -4.298284 \\
\hline $\mathrm{H}$ & 8.890674 & 9.688436 & -2.997277 \\
\hline $\mathrm{H}$ & 12.190476 & 7.109465 & 1.351499 \\
\hline $\mathrm{H}$ & 11.113599 & 6.514460 & -0.814594 \\
\hline $\mathrm{H}$ & 10.536336 & 10.687010 & -1.658897 \\
\hline $\mathrm{H}$ & 11.583283 & 11.267746 & 0.485547 \\
\hline $\mathrm{H}$ & 14.417485 & 10.279745 & 0.467709 \\
\hline $\mathrm{H}$ & 16.008058 & 12.143620 & 0.625437 \\
\hline $\mathrm{H}$ & 14.432702 & 13.160386 & 4.498483 \\
\hline $\mathrm{H}$ & 12.818310 & 11.283015 & 4.322019 \\
\hline $\mathrm{H}$ & 17.814096 & 13.273656 & 1.611044 \\
\hline $\mathrm{H}$ & 16.647379 & 14.372372 & 0.810872 \\
\hline $\mathrm{H}$ & 17.703203 & 14.983643 & 2.113103 \\
\hline
\end{tabular}




\begin{tabular}{|c|c|c|c|}
\hline $\mathrm{H}$ & 16.086612 & 11.688781 & 5.983412 \\
\hline $\mathrm{H}$ & 16.886827 & 13.801865 & 5.035545 \\
\hline $\mathrm{H}$ & 17.912808 & 15.181080 & 8.982912 \\
\hline $\mathrm{H}$ & 17.118896 & 13.018811 & 9.931178 \\
\hline $\mathrm{H}$ & 10.786105 & 11.952334 & 12.117038 \\
\hline $\mathrm{H}$ & 10.862563 & 11.532637 & 13.851371 \\
\hline $\mathrm{H}$ & 11.952106 & 12.784389 & 13.191454 \\
\hline $\mathrm{H}$ & 12.627736 & 12.840529 & 10.959251 \\
\hline $\mathrm{H}$ & 14.101387 & 9.004330 & 12.214937 \\
\hline $\mathrm{H}$ & 15.764783 & 9.049432 & 10.362156 \\
\hline $\mathrm{H}$ & 14.249623 & 12.867482 & 9.120140 \\
\hline $\mathrm{H}$ & 10.507837 & 4.122329 & -0.808785 \\
\hline $\mathrm{H}$ & 8.886283 & 3.669191 & -0.219051 \\
\hline $\mathrm{H}$ & 9.871095 & 4.838446 & 0.704211 \\
\hline $\mathrm{H}$ & 11.406647 & 0.952595 & 2.204778 \\
\hline $\mathrm{H}$ & 12.201081 & 4.870569 & 0.620579 \\
\hline $\mathrm{H}$ & 14.421683 & 4.847942 & 1.675316 \\
\hline $\mathrm{H}$ & 13.651709 & 0.946643 & 3.283250 \\
\hline $\mathrm{H}$ & 11.098713 & -1.699859 & 10.998940 \\
\hline $\mathrm{H}$ & 9.754820 & -1.944022 & 12.149342 \\
\hline $\mathrm{H}$ & 9.543449 & -0.846442 & 10.756559 \\
\hline $\mathrm{H}$ & 12.555290 & 2.222669 & 8.916213 \\
\hline $\mathrm{H}$ & 11.325849 & 0.254819 & 9.727878 \\
\hline $\mathrm{H}$ & 12.775123 & 3.772440 & 12.910531 \\
\hline $\mathrm{H}$ & 11.560155 & 1.767801 & 13.747687 \\
\hline $\mathrm{H}$ & 19.187750 & 7.408035 & 13.936790 \\
\hline $\mathrm{H}$ & 18.333758 & 7.906057 & 12.448964 \\
\hline $\mathrm{H}$ & 17.450333 & 7.815203 & 14.003037 \\
\hline $\mathrm{H}$ & 16.043971 & 7.613611 & 12.169144 \\
\hline $\mathrm{H}$ & 14.061974 & 6.812343 & 10.953063 \\
\hline $\mathrm{H}$ & 17.399244 & 3.563084 & 12.643260 \\
\hline $\mathrm{H}$ & 15.403057 & 2.759443 & 11.393804 \\
\hline $\mathrm{H}$ & 10.931367 & 4.644740 & 9.588448 \\
\hline $\mathrm{H}$ & 10.170136 & 5.848280 & 7.566565 \\
\hline $\mathrm{H}$ & 15.043741 & 5.390915 & 8.542254 \\
\hline $\mathrm{H}$ & 9.926645 & 7.288738 & 5.283295 \\
\hline $\mathrm{H}$ & 10.261660 & 8.720936 & 3.281436 \\
\hline $\mathrm{H}$ & 14.527874 & 8.553645 & 3.812145 \\
\hline $\mathrm{H}$ & 18.649309 & 5.828806 & 4.764327 \\
\hline $\mathrm{H}$ & 17.948206 & 3.850881 & 3.457101 \\
\hline $\mathrm{H}$ & 13.846873 & 4.480213 & 4.622162 \\
\hline $\mathrm{H}$ & 14.193834 & 9.228634 & 7.773642 \\
\hline $\mathrm{H}$ & 18.814373 & 8.082467 & 6.263816 \\
\hline $\mathrm{H}$ & 18.404254 & 10.100916 & 7.652351 \\
\hline
\end{tabular}


(b) spiro-MeOTAD ${ }^{+} \mathrm{TFSI}^{-}$

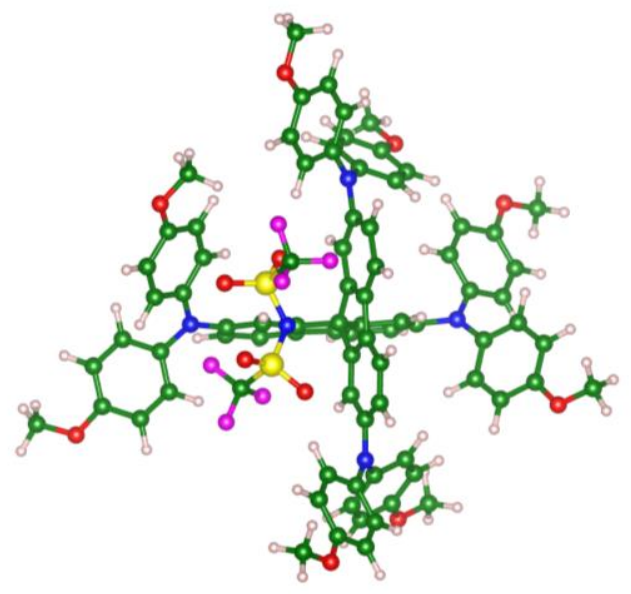

$\begin{array}{lrrr}\mathrm{O} & 13.206755 & 21.354689 & 12.267535 \\ \mathrm{O} & 5.287443 & 6.266258 & 19.362019 \\ \mathrm{O} & 11.353606 & 18.809669 & 8.552042 \\ \mathrm{~N} & 6.987904 & 15.330649 & 8.838763 \\ \mathrm{C} & 6.852539 & 12.489321 & 13.687951 \\ \mathrm{~N} & 10.980489 & 16.841986 & 14.723787 \\ \mathrm{O} & 5.794991 & 8.938959 & 5.935716 \\ \mathrm{O} & 7.690217 & 16.951849 & 19.251793 \\ \mathrm{~N} & 8.270136 & 10.360924 & 17.015658 \\ \mathrm{~N} & 9.881170 & 8.614841 & 9.718209 \\ \mathrm{C} & 5.515715 & 13.822279 & 11.903580 \\ \mathrm{C} & 8.219342 & 12.141828 & 13.798811 \\ \mathrm{C} & 7.907136 & 13.383477 & 11.816178 \\ \mathrm{C} & 9.954858 & 14.845251 & 13.756711 \\ \mathrm{C} & 10.102648 & 13.656216 & 13.067292 \\ \mathrm{C} & 9.812480 & 11.653631 & 11.834476 \\ \mathrm{C} & 11.100461 & 15.604599 & 14.045413 \\ \mathrm{C} & 8.698296 & 11.435031 & 14.876942 \\ \mathrm{C} & 9.346787 & 10.567966 & 11.114320 \\ \mathrm{C} & 9.007092 & 12.695456 & 12.611952 \\ \mathrm{O} & 13.288803 & 11.686187 & 19.081407 \\ \mathrm{C} & 11.369616 & 13.191040 & 12.662358 \\ \mathrm{C} & 12.672763 & 20.148156 & 14.333371 \\ \mathrm{C} & 12.115873 & 19.276705 & 12.144359 \\ \mathrm{C} & 5.953048 & 12.091701 & 14.684475 \\ \mathrm{C} & 11.193120 & 11.944021 & 11.925500 \\ \mathrm{C} & 6.872038 & 14.625947 & 10.052212\end{array}$




\begin{tabular}{|c|c|c|c|}
\hline $\mathrm{C}$ & 12.679703 & 20.284604 & 12.939785 \\
\hline $\mathrm{C}$ & 8.372051 & 17.123535 & 9.739534 \\
\hline $\mathrm{C}$ & 12.511520 & 13.945219 & 12.955366 \\
\hline $\mathrm{C}$ & 10.288958 & 9.726977 & 10.487764 \\
\hline $\mathrm{C}$ & 11.546854 & 18.001183 & 14.135171 \\
\hline $\mathrm{C}$ & 8.082616 & 16.224100 & 8.698341 \\
\hline $\mathrm{C}$ & 12.369979 & 15.146992 & 13.644731 \\
\hline $\mathrm{C}$ & 11.668658 & 9.989640 & 10.614305 \\
\hline $\mathrm{C}$ & 6.661622 & 13.251087 & 12.470278 \\
\hline $\mathrm{C}$ & 7.511795 & 9.313694 & 17.592561 \\
\hline $\mathrm{C}$ & 10.293806 & 17.947275 & 8.527456 \\
\hline $\mathrm{C}$ & 6.425119 & 11.384173 & 15.778485 \\
\hline $\mathrm{C}$ & 12.125219 & 11.094680 & 11.323253 \\
\hline $\mathrm{C}$ & 7.516775 & 9.104718 & 18.984056 \\
\hline $\mathrm{C}$ & 8.027090 & 14.062880 & 10.625903 \\
\hline $\mathrm{C}$ & 6.044430 & 15.146981 & 7.803286 \\
\hline $\mathrm{C}$ & 7.794061 & 11.055791 & 15.891776 \\
\hline $\mathrm{C}$ & 5.718587 & 16.195585 & 6.934747 \\
\hline $\mathrm{C}$ & 7.152295 & 7.683643 & 7.381874 \\
\hline $\mathrm{C}$ & 11.548654 & 18.156230 & 12.737056 \\
\hline $\mathrm{C}$ & 6.829834 & 8.928905 & 6.821362 \\
\hline $\mathrm{C}$ & 4.465456 & 13.727670 & 6.636745 \\
\hline $\mathrm{C}$ & 8.867748 & 8.724517 & 8.742374 \\
\hline $\mathrm{C}$ & 5.624468 & 14.501027 & 10.698730 \\
\hline $\mathrm{C}$ & 9.464295 & 17.972739 & 9.658908 \\
\hline $\mathrm{C}$ & 5.411565 & 13.898265 & 7.635874 \\
\hline $\mathrm{C}$ & 9.534286 & 10.712188 & 17.553368 \\
\hline $\mathrm{C}$ & 10.001635 & 17.072077 & 7.473328 \\
\hline $\mathrm{C}$ & 7.547091 & 10.066352 & 7.206644 \\
\hline $\mathrm{C}$ & 9.879496 & 12.060262 & 17.706071 \\
\hline $\mathrm{C}$ & 10.460437 & 9.724192 & 17.928946 \\
\hline $\mathrm{C}$ & 6.772306 & 8.074767 & 19.539112 \\
\hline $\mathrm{C}$ & 4.148963 & 14.776913 & 5.760679 \\
\hline $\mathrm{C}$ & 10.140272 & 16.920043 & 15.860484 \\
\hline $\mathrm{C}$ & 5.973809 & 7.452551 & 17.335496 \\
\hline $\mathrm{C}$ & 6.739392 & 8.474612 & 16.779466 \\
\hline $\mathrm{C}$ & 9.300407 & 18.014818 & 16.086691 \\
\hline $\mathrm{C}$ & 12.045467 & 11.435526 & 18.580473 \\
\hline $\mathrm{C}$ & 8.464593 & 17.005859 & 18.125234 \\
\hline $\mathrm{C}$ & 5.991091 & 7.241677 & 18.720345 \\
\hline $\mathrm{C}$ & 8.902672 & 16.212675 & 7.567272 \\
\hline $\mathrm{C}$ & 12.119366 & 19.006540 & 14.920268 \\
\hline $\mathrm{C}$ & 4.787407 & 16.013978 & 5.909760 \\
\hline $\mathrm{C}$ & 9.298025 & 15.900022 & 17.900123 \\
\hline
\end{tabular}




\begin{tabular}{|c|c|c|c|}
\hline $\mathrm{C}$ & 8.565284 & 9.958405 & 8.149119 \\
\hline $\mathrm{C}$ & 11.699057 & 10.081795 & 18.441477 \\
\hline $\mathrm{C}$ & 11.127473 & 12.426514 & 18.208875 \\
\hline $\mathrm{C}$ & 8.474796 & 18.067611 & 17.214371 \\
\hline $\mathrm{C}$ & 8.141802 & 7.584567 & 8.341384 \\
\hline $\mathrm{C}$ & 10.129485 & 15.863303 & 16.790334 \\
\hline $\mathrm{C}$ & 12.243256 & 18.816719 & 7.442691 \\
\hline $\mathrm{C}$ & 13.696024 & 13.042718 & 19.218426 \\
\hline $\mathrm{C}$ & 5.179503 & 10.193474 & 5.661711 \\
\hline $\mathrm{C}$ & 4.465141 & 5.407344 & 18.577842 \\
\hline $\mathrm{C}$ & 6.802160 & 18.031563 & 19.503709 \\
\hline $\mathrm{C}$ & 10.611565 & 7.406214 & 9.863887 \\
\hline $\mathrm{C}$ & 11.110852 & 6.719233 & 8.753759 \\
\hline $\mathrm{C}$ & 10.850855 & 6.891945 & 11.149795 \\
\hline $\mathrm{C}$ & 11.829088 & 5.532224 & 8.911783 \\
\hline $\mathrm{H}$ & 10.931346 & 7.112172 & 7.758964 \\
\hline $\mathrm{C}$ & 11.576289 & 5.722258 & 11.316085 \\
\hline $\mathrm{H}$ & 10.455416 & 7.417833 & 12.011968 \\
\hline $\mathrm{C}$ & 12.067785 & 5.030211 & 10.197704 \\
\hline $\mathrm{H}$ & 12.201331 & 5.021719 & 8.032411 \\
\hline $\mathrm{H}$ & 11.758428 & 5.313047 & 12.303845 \\
\hline $\mathrm{O}$ & 12.759868 & 3.884062 & 10.464087 \\
\hline $\mathrm{C}$ & 13.259577 & 3.129951 & 9.367453 \\
\hline $\mathrm{H}$ & 13.983206 & 3.710342 & 8.781553 \\
\hline $\mathrm{H}$ & 13.757539 & 2.263859 & 9.803966 \\
\hline $\mathrm{H}$ & 12.446068 & 2.792966 & 8.713162 \\
\hline $\mathrm{C}$ & 13.789163 & 22.401286 & 13.030047 \\
\hline $\mathrm{H}$ & 14.136949 & 23.140326 & 12.307514 \\
\hline $\mathrm{H}$ & 13.053590 & 22.864826 & 13.699940 \\
\hline $\mathrm{H}$ & 14.640907 & 22.039730 & 13.620318 \\
\hline $\mathrm{O}$ & 3.216049 & 14.494722 & 4.802425 \\
\hline $\mathrm{C}$ & 2.845047 & 15.529448 & 3.902598 \\
\hline $\mathrm{H}$ & 3.704151 & 15.870698 & 3.311018 \\
\hline $\mathrm{H}$ & 2.097516 & 15.094271 & 3.238790 \\
\hline $\mathrm{H}$ & 2.407375 & 16.382882 & 4.435656 \\
\hline $\mathrm{H}$ & 5.653159 & 13.071265 & 8.296004 \\
\hline $\mathrm{H}$ & 3.957079 & 12.778059 & 6.515645 \\
\hline $\mathrm{H}$ & 4.559937 & 16.845936 & 5.254872 \\
\hline $\mathrm{H}$ & 6.193434 & 17.162487 & 7.059250 \\
\hline $\mathrm{H}$ & 8.687156 & 15.521893 & 6.758954 \\
\hline $\mathrm{H}$ & 10.625003 & 17.033165 & 6.588669 \\
\hline $\mathrm{H}$ & 9.688794 & 18.669763 & 10.458226 \\
\hline $\mathrm{H}$ & 7.734627 & 17.139955 & 10.616921 \\
\hline $\mathrm{H}$ & 12.717085 & 17.836588 & 7.308013 \\
\hline
\end{tabular}




\begin{tabular}{|c|c|c|c|}
\hline $\mathrm{H}$ & 11.724502 & 19.102762 & 6.519368 \\
\hline $\mathrm{H}$ & 13.005079 & 19.560476 & 7.677138 \\
\hline $\mathrm{H}$ & 11.107437 & 17.386195 & 12.114155 \\
\hline $\mathrm{H}$ & 12.116255 & 19.391055 & 11.065048 \\
\hline $\mathrm{H}$ & 13.104224 & 20.908322 & 14.972892 \\
\hline $\mathrm{H}$ & 12.127010 & 18.901841 & 16.000042 \\
\hline $\mathrm{H}$ & 6.071047 & 18.143324 & 18.693132 \\
\hline $\mathrm{H}$ & 6.282582 & 17.781584 & 20.429132 \\
\hline $\mathrm{H}$ & 7.347665 & 18.974853 & 19.635041 \\
\hline $\mathrm{H}$ & 7.838197 & 18.932327 & 17.355606 \\
\hline $\mathrm{H}$ & 9.282980 & 15.084602 & 18.615907 \\
\hline $\mathrm{H}$ & 10.768947 & 15.004264 & 16.619578 \\
\hline $\mathrm{H}$ & 9.289331 & 18.836456 & 15.379100 \\
\hline $\mathrm{H}$ & 5.841184 & 10.853737 & 5.087750 \\
\hline $\mathrm{H}$ & 4.291980 & 9.969836 & 5.068990 \\
\hline $\mathrm{H}$ & 4.889149 & 10.684990 & 6.595659 \\
\hline $\mathrm{H}$ & 6.560154 & 6.819424 & 7.103330 \\
\hline $\mathrm{H}$ & 7.307844 & 11.042497 & 6.804718 \\
\hline $\mathrm{H}$ & 9.108110 & 10.848031 & 8.443718 \\
\hline $\mathrm{H}$ & 8.336073 & 6.630078 & 8.814874 \\
\hline $\mathrm{H}$ & 5.062120 & 4.838124 & 17.855384 \\
\hline $\mathrm{H}$ & 3.994919 & 4.722088 & 19.283316 \\
\hline $\mathrm{H}$ & 3.692895 & 5.975174 & 18.045688 \\
\hline $\mathrm{H}$ & 6.703451 & 8.630560 & 15.709233 \\
\hline $\mathrm{H}$ & 5.379641 & 6.836791 & 16.672478 \\
\hline $\mathrm{H}$ & 8.101633 & 9.755796 & 19.624298 \\
\hline $\mathrm{H}$ & 6.765548 & 7.906628 & 20.610621 \\
\hline $\mathrm{H}$ & 14.710895 & 13.009628 & 19.614962 \\
\hline $\mathrm{H}$ & 13.697297 & 13.556390 & 18.249218 \\
\hline $\mathrm{H}$ & 13.047602 & 13.584105 & 19.918118 \\
\hline $\mathrm{H}$ & 11.364612 & 13.477047 & 18.318276 \\
\hline $\mathrm{H}$ & 9.168593 & 12.827676 & 17.420186 \\
\hline $\mathrm{H}$ & 12.423988 & 9.327070 & 18.726434 \\
\hline $\mathrm{H}$ & 10.201848 & 8.677848 & 17.809064 \\
\hline $\mathrm{H}$ & 5.741404 & 11.068819 & 16.557136 \\
\hline $\mathrm{H}$ & 4.896926 & 12.324226 & 14.604573 \\
\hline $\mathrm{H}$ & 9.746064 & 11.166001 & 14.951659 \\
\hline $\mathrm{H}$ & 4.551740 & 13.729423 & 12.390743 \\
\hline $\mathrm{H}$ & 4.747606 & 14.942050 & 10.238257 \\
\hline $\mathrm{H}$ & 8.983925 & 14.155713 & 10.123678 \\
\hline $\mathrm{H}$ & 13.189714 & 11.300274 & 11.383844 \\
\hline $\mathrm{H}$ & 12.372774 & 9.325748 & 10.125518 \\
\hline $\mathrm{H}$ & 8.285033 & 10.371037 & 11.027243 \\
\hline $\mathrm{H}$ & 8.975349 & 15.201707 & 14.058455 \\
\hline
\end{tabular}




$\begin{array}{lrrr}\mathrm{H} & 13.497521 & 13.604840 & 12.653303 \\ \mathrm{H} & 13.238821 & 15.754002 & 13.876542 \\ \mathrm{~N} & 5.597625 & 10.038732 & 11.833360 \\ \mathrm{~S} & 5.298141 & 8.671700 & 12.653688 \\ \mathrm{~S} & 5.276074 & 10.206065 & 10.258146 \\ \mathrm{O} & 5.252373 & 8.988770 & 9.447165 \\ \mathrm{O} & 6.039493 & 11.369704 & 9.785778 \\ \mathrm{O} & 4.388050 & 7.708177 & 12.033766 \\ \mathrm{O} & 5.127144 & 9.020617 & 14.069660 \\ \mathrm{C} & 3.489074 & 10.804831 & 10.209635 \\ \mathrm{C} & 6.979383 & 7.821946 & 12.637902 \\ \mathrm{~F} & 2.647882 & 9.859827 & 10.639037 \\ \mathrm{~F} & 3.171834 & 11.118245 & 8.935026 \\ \mathrm{~F} & 3.309831 & 11.906318 & 10.961955 \\ \mathrm{~F} & 6.902731 & 6.658467 & 13.310192 \\ \mathrm{~F} & 7.388605 & 7.566758 & 11.388084 \\ \mathrm{~F} & 7.913302 & 8.586477 & 13.239971\end{array}$

(c) $\mathrm{Zn}^{+}(t \mathrm{BP})_{4}$

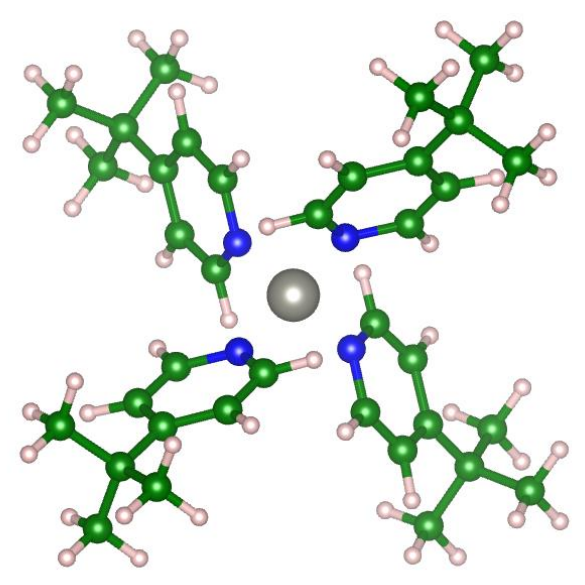

$\begin{array}{rrrr}\mathrm{Zn} & 0.30060500 & -1.49465500 & 0.03594300 \\ \mathrm{C} & 3.13422900 & -2.26533400 & 0.07673700 \\ \mathrm{C} & 1.99425100 & -2.98302800 & 1.97011400 \\ \mathrm{C} & 4.30784500 & -2.86301400 & 0.51989400 \\ \mathrm{H} & 3.09754900 & -1.73680800 & -0.87003200 \\ \mathrm{C} & 3.12615000 & -3.59955300 & 2.46068700 \\ \mathrm{H} & 1.05528700 & -3.01767100 & 2.51379300 \\ \mathrm{H} & 5.18863000 & -2.78091200 & -0.10441100 \\ \mathrm{H} & 3.06136100 & -4.11720000 & 3.41057200 \\ \mathrm{~N} & 1.97661200 & -2.30728500 & 0.79187800 \\ \mathrm{C} & 4.34539000 & -3.55445800 & 1.73808500 \\ \mathrm{C} & 5.59636100 & -4.23480700 & 2.28793100\end{array}$




\begin{tabular}{|c|c|c|c|}
\hline $\mathrm{C}$ & 5.94293000 & -3.61574700 & 3.66277200 \\
\hline $\mathrm{C}$ & 5.31393200 & -5.74606300 & 2.46048100 \\
\hline $\mathrm{C}$ & 6.80594800 & -4.06468600 & 1.35386900 \\
\hline $\mathrm{H}$ & 6.14634600 & -2.54404300 & 3.56971000 \\
\hline $\mathrm{H}$ & 5.13175200 & -3.74598600 & 4.38510600 \\
\hline $\mathrm{H}$ & 6.83551200 & -4.09821700 & 4.07361500 \\
\hline $\mathrm{H}$ & 5.06441400 & -6.20996700 & 1.50069500 \\
\hline $\mathrm{H}$ & 6.20091000 & -6.24700800 & 2.86126000 \\
\hline $\mathrm{H}$ & 4.48651300 & -5.93014100 & 3.15203500 \\
\hline $\mathrm{H}$ & 7.67570200 & -4.56471500 & 1.78941100 \\
\hline $\mathrm{H}$ & 6.62778600 & -4.51257700 & 0.37054600 \\
\hline $\mathrm{H}$ & 7.06824900 & -3.01061500 & 1.21471600 \\
\hline $\mathrm{C}$ & 0.84970500 & -3.05515000 & -2.39109000 \\
\hline $\mathrm{C}$ & -0.02684000 & -0.95648500 & -2.86712400 \\
\hline $\mathrm{C}$ & 0.96174700 & -3.34865600 & -3.74477200 \\
\hline $\mathrm{H}$ & 1.16270500 & -3.76800500 & -1.63550400 \\
\hline $\mathrm{C}$ & 0.06350900 & -1.18779700 & -4.22364900 \\
\hline $\mathrm{H}$ & -0.40529200 & -0.01472500 & -2.48206300 \\
\hline $\mathrm{H}$ & 1.36499100 & -4.31358400 & -4.02518900 \\
\hline $\mathrm{H}$ & -0.25990300 & -0.40775300 & -4.90290600 \\
\hline $\mathrm{N}$ & 0.34982200 & -1.87279200 & -1.93775100 \\
\hline $\mathrm{C}$ & 0.56694700 & -2.41874600 & -4.71575000 \\
\hline $\mathrm{C}$ & 0.66057400 & -2.67091000 & -6.21832500 \\
\hline $\mathrm{C}$ & 1.22634700 & -4.06462600 & -6.53746800 \\
\hline $\mathrm{C}$ & -0.75297900 & -2.55809800 & -6.83689200 \\
\hline $\mathrm{C}$ & 1.58595500 & -1.60489300 & -6.85138000 \\
\hline $\mathrm{H}$ & 2.24199000 & -4.19125000 & -6.14798600 \\
\hline $\mathrm{H}$ & 0.59539100 & -4.86288500 & -6.13259500 \\
\hline $\mathrm{H}$ & 1.27159500 & -4.19988700 & -7.62184500 \\
\hline $\mathrm{H}$ & -1.19026600 & -1.56800600 & -6.67798000 \\
\hline $\mathrm{H}$ & -0.70129100 & -2.72995100 & -7.91673500 \\
\hline $\mathrm{H}$ & -1.43010200 & -3.30201700 & -6.40482800 \\
\hline $\mathrm{H}$ & 1.65763300 & -1.76833500 & -7.93140600 \\
\hline $\mathrm{H}$ & 1.20824200 & -0.59059400 & -6.69231200 \\
\hline $\mathrm{H}$ & 2.59474800 & -1.66195500 & -6.42980300 \\
\hline $\mathrm{C}$ & -0.98464300 & 1.12359500 & 0.37634100 \\
\hline $\mathrm{C}$ & 1.30638200 & 1.20617800 & 0.75606000 \\
\hline $\mathrm{C}$ & -1.11364000 & 2.47413900 & 0.67693700 \\
\hline $\mathrm{H}$ & -1.84848200 & 0.52436000 & 0.10841600 \\
\hline $\mathrm{C}$ & 1.24153900 & 2.54781200 & 1.06855500 \\
\hline $\mathrm{H}$ & 2.24825500 & 0.66727200 & 0.78585400 \\
\hline $\mathrm{H}$ & -2.10171200 & 2.91439600 & 0.62992500 \\
\hline $\mathrm{H}$ & 2.15760400 & 3.05934400 & 1.33979100 \\
\hline $\mathrm{N}$ & 0.21473800 & 0.47981500 & 0.40104100 \\
\hline
\end{tabular}




\begin{tabular}{|c|c|c|c|}
\hline $\mathrm{C}$ & 0.00430200 & 3.23989900 & 1.03377700 \\
\hline $\mathrm{C}$ & -0.06100100 & 4.72606700 & 1.37598500 \\
\hline $\mathrm{C}$ & -1.49102900 & 5.28170900 & 1.27188300 \\
\hline $\mathrm{C}$ & 0.44542600 & 4.93305400 & 2.82309500 \\
\hline $\mathrm{C}$ & 0.84546000 & 5.50868600 & 0.39654800 \\
\hline $\mathrm{H}$ & -1.89336900 & 5.18378400 & 0.25808900 \\
\hline $\mathrm{H}$ & -2.17378900 & 4.78299700 & 1.96786600 \\
\hline $\mathrm{H}$ & -1.48739200 & 6.34637700 & 1.52224000 \\
\hline $\mathrm{H}$ & 1.47828000 & 4.59421600 & 2.94626900 \\
\hline $\mathrm{H}$ & 0.41046000 & 5.99633700 & 3.08101600 \\
\hline $\mathrm{H}$ & -0.17886000 & 4.38766000 & 3.53826400 \\
\hline $\mathrm{H}$ & 0.81384100 & 6.57694800 & 0.63335200 \\
\hline $\mathrm{H}$ & 1.88853000 & 5.18475800 & 0.45833800 \\
\hline $\mathrm{H}$ & 0.50967000 & 5.37803800 & -0.63726100 \\
\hline $\mathrm{C}$ & -2.07127700 & -3.26424100 & 0.29824300 \\
\hline $\mathrm{C}$ & -1.80640100 & -1.77646200 & 2.06326600 \\
\hline $\mathrm{C}$ & -3.23271700 & -3.76470200 & 0.84835300 \\
\hline $\mathrm{H}$ & -1.69036600 & -3.63914000 & -0.64663000 \\
\hline $\mathrm{C}$ & -2.96750600 & -2.24133900 & 2.66892500 \\
\hline $\mathrm{H}$ & -1.22069800 & -0.98074500 & 2.51132700 \\
\hline $\mathrm{H}$ & -3.75771400 & -4.55090400 & 0.31854200 \\
\hline $\mathrm{H}$ & -3.26898000 & -1.79057500 & 3.60606600 \\
\hline $\mathrm{C}$ & -3.72549100 & -3.26164100 & 2.07908200 \\
\hline $\mathrm{N}$ & -1.34135900 & -2.28232900 & 0.88817700 \\
\hline $\mathrm{C}$ & -5.00866700 & -3.82289000 & 2.68624800 \\
\hline $\mathrm{C}$ & -6.17409300 & -3.60611700 & 1.69218100 \\
\hline $\mathrm{C}$ & -4.82409200 & -5.33771900 & 2.94067800 \\
\hline $\mathrm{C}$ & -5.36548500 & -3.14125100 & 4.01760100 \\
\hline $\mathrm{H}$ & -6.32577200 & -2.54034000 & 1.49281300 \\
\hline $\mathrm{H}$ & -5.99444100 & -4.10812100 & 0.73695500 \\
\hline $\mathrm{H}$ & -7.10095500 & -4.01011200 & 2.11170900 \\
\hline $\mathrm{H}$ & -4.00279300 & -5.51992900 & 3.64128500 \\
\hline $\mathrm{H}$ & -5.73921000 & -5.75684200 & 3.3709830 \\
\hline $\mathrm{H}$ & -4.61007700 & -5.88352100 & 2.01707500 \\
\hline $\mathrm{H}$ & -6.28761800 & -3.57587800 & 4.4138190 \\
\hline $\mathrm{H}$ & -4.58408500 & -3.28499300 & 4.77125400 \\
\hline $\mathrm{H}$ & -5.53603200 & -2.06676200 & 3.8924800 \\
\hline
\end{tabular}

(d) $\mathrm{Zn}^{+}(t \mathrm{BP})_{3} \mathrm{TFSI}^{-}$ 


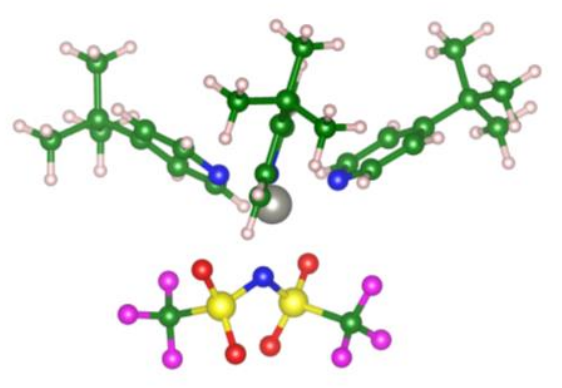

\begin{tabular}{|c|c|c|c|}
\hline $\mathrm{Zn}$ & 0.182284 & -1.062617 & 0.034729 \\
\hline $\mathrm{C}$ & 2.942630 & -1.986195 & -0.283652 \\
\hline $\mathrm{C}$ & 2.131986 & -2.438583 & 1.846205 \\
\hline $\mathrm{C}$ & 4.094593 & -2.720546 & -0.032636 \\
\hline $\mathrm{H}$ & 2.796500 & -1.489098 & -1.234503 \\
\hline $\mathrm{C}$ & 3.243988 & -3.193685 & 2.147487 \\
\hline $\mathrm{H}$ & 1.349795 & -2.287639 & 2.579065 \\
\hline $\mathrm{H}$ & 4.833710 & -2.784317 & -0.821805 \\
\hline $\mathrm{H}$ & 3.307705 & -3.643175 & 3.132106 \\
\hline $\mathrm{N}$ & 1.956898 & -1.820427 & 0.646825 \\
\hline $\mathrm{C}$ & 4.288955 & -3.358174 & 1.199148 \\
\hline $\mathrm{C}$ & 5.524745 & -4.185274 & 1.544424 \\
\hline $\mathrm{C}$ & 6.227290 & -3.563270 & 2.774112 \\
\hline $\mathrm{C}$ & 5.093119 & -5.632038 & 1.880633 \\
\hline $\mathrm{C}$ & 6.531435 & -4.235542 & 0.382844 \\
\hline $\mathrm{H}$ & 6.547640 & -2.538353 & 2.560869 \\
\hline $\mathrm{H}$ & 5.566566 & -3.533918 & 3.645523 \\
\hline $\mathrm{H}$ & 7.111724 & -4.152013 & 3.042411 \\
\hline $\mathrm{H}$ & 4.597067 & -6.098623 & 1.023041 \\
\hline $\mathrm{H}$ & 5.968322 & -6.237274 & 2.142457 \\
\hline $\mathrm{H}$ & 4.400277 & -5.660433 & 2.726900 \\
\hline $\mathrm{H}$ & 7.398687 & -4.836635 & 0.674126 \\
\hline $\mathrm{H}$ & 6.095456 & -4.693199 & -0.511533 \\
\hline $\mathrm{H}$ & 6.893813 & -3.236709 & 0.118047 \\
\hline $\mathrm{C}$ & 0.641273 & -2.946403 & -2.082140 \\
\hline $\mathrm{C}$ & 0.062150 & -0.874451 & -2.986701 \\
\hline $\mathrm{C}$ & 0.883680 & -3.463326 & -3.346402 \\
\hline $\mathrm{H}$ & 0.769807 & -3.559037 & -1.197091 \\
\hline $\mathrm{C}$ & 0.308966 & -1.331580 & -4.260838 \\
\hline $\mathrm{H}$ & -0.288913 & 0.133478 & -2.807044 \\
\hline $\mathrm{H}$ & 1.206199 & -4.495611 & -3.413886 \\
\hline $\mathrm{H}$ & 0.160971 & -0.642829 & -5.085184 \\
\hline $\mathrm{N}$ & 0.208661 & -1.657342 & -1.876061 \\
\hline $\mathrm{C}$ & 0.732659 & -2.672088 & -4.494196 \\
\hline
\end{tabular}




\begin{tabular}{|c|c|c|c|}
\hline $\mathrm{C}$ & 1.004177 & -3.163605 & -5.912833 \\
\hline $\mathrm{C}$ & 1.445479 & -4.636642 & -5.940915 \\
\hline $\mathrm{C}$ & -0.282363 & -3.023223 & -6.760351 \\
\hline $\mathrm{C}$ & 2.125365 & -2.305761 & -6.545916 \\
\hline $\mathrm{H}$ & 2.373789 & -4.791800 & -5.380825 \\
\hline $\mathrm{H}$ & 0.677048 & -5.296938 & -5.524838 \\
\hline $\mathrm{H}$ & 1.626064 & -4.947401 & -6.974994 \\
\hline $\mathrm{H}$ & -0.627175 & -1.985861 & -6.801919 \\
\hline $\mathrm{H}$ & -0.098970 & -3.357861 & -7.787775 \\
\hline $\mathrm{H}$ & -1.091398 & -3.629876 & -6.340185 \\
\hline $\mathrm{H}$ & 2.324190 & -2.636319 & -7.571818 \\
\hline $\mathrm{H}$ & 1.851524 & -1.247169 & -6.581242 \\
\hline $\mathrm{H}$ & 3.052831 & -2.394120 & -5.970410 \\
\hline $\mathrm{N}$ & -0.107206 & 0.871738 & 0.691800 \\
\hline $\mathrm{C}$ & -2.303798 & -2.653182 & 0.198712 \\
\hline $\mathrm{C}$ & -1.557990 & -1.953290 & 2.278190 \\
\hline $\mathrm{C}$ & -3.417136 & -3.250868 & 0.758876 \\
\hline $\mathrm{H}$ & -2.130057 & -2.665015 & -0.872372 \\
\hline $\mathrm{C}$ & -2.653938 & -2.535054 & 2.903703 \\
\hline $\mathrm{H}$ & -0.816979 & -1.397708 & 2.842702 \\
\hline $\mathrm{H}$ & -4.124898 & -3.742668 & 0.101500 \\
\hline $\mathrm{H}$ & -2.735821 & -2.440038 & 3.978988 \\
\hline $\mathrm{C}$ & -3.628808 & -3.206992 & 2.154835 \\
\hline $\mathrm{N}$ & -1.371749 & -2.011851 & 0.939700 \\
\hline $\mathrm{C}$ & -4.869296 & -3.853274 & 2.769854 \\
\hline $\mathrm{C}$ & -6.130954 & -3.182620 & 2.177782 \\
\hline $\mathrm{C}$ & -4.877964 & -5.361060 & 2.426918 \\
\hline $\mathrm{C}$ & -4.905428 & -3.699043 & 4.299541 \\
\hline $\mathrm{H}$ & -6.145253 & -2.111499 & 2.403134 \\
\hline $\mathrm{H}$ & -6.180656 & -3.299102 & 1.091263 \\
\hline $\mathrm{H}$ & -7.031438 & -3.636017 & 2.605873 \\
\hline $\mathrm{H}$ & -3.991862 & -5.859032 & 2.833997 \\
\hline $\mathrm{H}$ & -5.766217 & -5.837166 & 2.855828 \\
\hline $\mathrm{H}$ & -4.895360 & -5.531915 & 1.346575 \\
\hline $\mathrm{H}$ & -5.806644 & -4.178816 & 4.693386 \\
\hline $\mathrm{H}$ & -4.041762 & -4.174477 & 4.776264 \\
\hline $\mathrm{H}$ & -4.932376 & -2.646867 & 4.601119 \\
\hline $\mathrm{S}$ & 0.637325 & 1.406048 & 2.065887 \\
\hline $\mathrm{S}$ & -1.214449 & 1.799543 & -0.123699 \\
\hline $\mathrm{O}$ & -0.784956 & 3.169919 & -0.368494 \\
\hline $\mathrm{O}$ & -0.051205 & 2.538215 & 2.676340 \\
\hline $\mathrm{O}$ & 0.980369 & 0.214862 & 2.846325 \\
\hline $\mathrm{O}$ & -1.662168 & 0.928522 & -1.215446 \\
\hline $\mathrm{C}$ & -2.735922 & 1.918342 & 1.014963 \\
\hline
\end{tabular}




$\begin{array}{lrrr}\text { C } & 2.282977 & 2.068602 & 1.431878 \\ \text { F } & -2.697495 & 3.007561 & 1.772790 \\ F & -2.818593 & 0.830037 & 1.797034 \\ \text { F } & -3.821954 & 1.968185 & 0.232143 \\ \text { F } & 3.044420 & 2.404350 & 2.480053 \\ F & 2.085568 & 3.140388 & 0.664327 \\ \text { F } & 2.914327 & 1.127622 & 0.715936\end{array}$

(e) $\mathrm{Zn}^{+}(t \mathrm{BP})_{2} \mathrm{TFSI}^{-}$

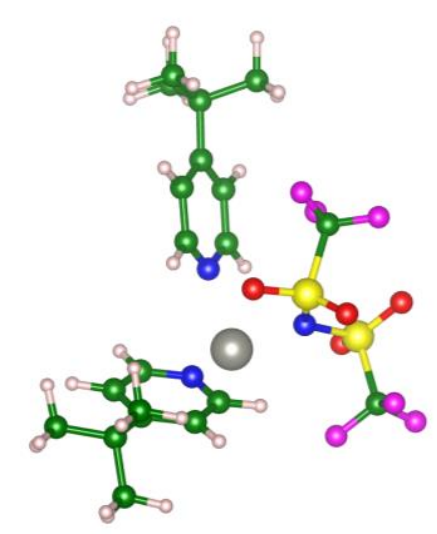

$\begin{array}{cccc}\text { Zn } & 0.83412400 & -0.68048600 & 0.05050200 \\ \mathrm{~N} & -0.82592400 & -2.14207200 & 0.38956900 \\ \mathrm{~S} & -0.72836100 & -3.77407600 & 0.16342200 \\ \mathrm{~S} & -2.25027100 & -1.42597500 & 0.80889000 \\ \mathrm{O} & -1.33569700 & -4.57226600 & 1.22560500 \\ \mathrm{O} & -3.42304100 & -2.23280900 & 0.47777400 \\ \mathrm{O} & 0.65752500 & -4.02814600 & -0.24477000 \\ \mathrm{O} & -2.14711100 & -0.02348700 & 0.40224300 \\ \mathrm{C} & -1.74328500 & -4.15335300 & -1.40943200 \\ \mathrm{C} & -2.16990100 & -1.37239100 & 2.69180400 \\ \mathrm{~F} & -1.83232000 & -3.06577900 & -2.19279200 \\ \mathrm{~F} & -2.96328000 & -4.58536100 & -1.11210500 \\ \mathrm{~F} & -1.10014900 & -5.11381000 & -2.09636400 \\ \mathrm{~F} & -2.27064000 & -2.59659900 & 3.21360200 \\ \mathrm{~F} & -3.17053300 & -0.61283400 & 3.15286600 \\ \mathrm{~F} & -0.99982700 & -0.83384600 & 3.08404900 \\ \mathrm{C} & 3.63601200 & -1.89621500 & 0.43721800 \\ \mathrm{C} & 2.12825500 & -2.61778800 & 2.01939000 \\ \mathrm{C} & 4.66124300 & -2.64355100 & 1.01030800 \\ \mathrm{H} & 3.81665600 & -1.28212200 & -0.44125000 \\ \mathrm{C} & 3.09745400 & -3.38184500 & 2.65008900\end{array}$




\begin{tabular}{|c|c|c|c|}
\hline $\mathrm{H}$ & 1.10867200 & -2.58801200 & 2.38767700 \\
\hline $\mathrm{H}$ & 5.64347600 & -2.60873700 & 0.55656800 \\
\hline $\mathrm{H}$ & 2.81469700 & -3.95534100 & 3.52528900 \\
\hline $\mathrm{N}$ & 2.38682200 & -1.88511700 & 0.92179600 \\
\hline $\mathrm{C}$ & 4.40911500 & -3.42078900 & 2.14845600 \\
\hline $\mathrm{C}$ & 5.47396300 & -4.28030300 & 2.83223700 \\
\hline $\mathrm{C}$ & 5.63321600 & -3.81463700 & 4.29767300 \\
\hline $\mathrm{C}$ & 5.01497900 & -5.75654900 & 2.80417300 \\
\hline $\mathrm{C}$ & 6.84017700 & -4.17720600 & 2.13391600 \\
\hline $\mathrm{H}$ & 5.94834000 & -2.76709000 & 4.34363300 \\
\hline $\mathrm{H}$ & 4.69985100 & -3.91202200 & 4.85928200 \\
\hline $\mathrm{H}$ & 6.39204400 & -4.42304600 & 4.80085200 \\
\hline $\mathrm{H}$ & 4.88414200 & -6.10621300 & 1.77512600 \\
\hline $\mathrm{H}$ & 5.76610300 & -6.38879100 & 3.28925500 \\
\hline $\mathrm{H}$ & 4.06659100 & -5.89764700 & 3.33000500 \\
\hline $\mathrm{H}$ & 7.56506300 & -4.80700700 & 2.65838800 \\
\hline $\mathrm{H}$ & 6.79342600 & -4.52252900 & 1.09580700 \\
\hline $\mathrm{H}$ & 7.22561800 & -3.15220100 & 2.14273900 \\
\hline $\mathrm{C}$ & 1.46915700 & -2.29504000 & -2.54126800 \\
\hline $\mathrm{C}$ & 0.01071600 & -0.53462500 & -2.83456400 \\
\hline $\mathrm{C}$ & 1.23471100 & -2.75592100 & -3.83364600 \\
\hline $\mathrm{H}$ & 2.12649200 & -2.83397300 & -1.86966600 \\
\hline $\mathrm{C}$ & -0.27125600 & -0.93276900 & -4.13224400 \\
\hline $\mathrm{H}$ & -0.47155200 & 0.33116400 & -2.38963800 \\
\hline $\mathrm{H}$ & 1.74191300 & -3.65502200 & -4.15866200 \\
\hline $\mathrm{H}$ & -0.98398900 & -0.35497100 & -4.70944700 \\
\hline $\mathrm{N}$ & 0.86629700 & -1.20649800 & -2.04220600 \\
\hline $\mathrm{C}$ & 0.33495400 & -2.08170100 & -4.66757900 \\
\hline $\mathrm{C}$ & -0.02103300 & -2.55939700 & -6.07636900 \\
\hline $\mathrm{C}$ & 0.77262800 & -3.81254000 & -6.48207100 \\
\hline $\mathrm{C}$ & -1.52970400 & -2.89847600 & -6.11072300 \\
\hline $\mathrm{C}$ & 0.28253600 & -1.43323100 & -7.08991700 \\
\hline $\mathrm{H}$ & 1.85212700 & -3.62763000 & -6.48249200 \\
\hline $\mathrm{H}$ & 0.56470200 & -4.65775000 & -5.81817800 \\
\hline $\mathrm{H}$ & 0.48735000 & -4.11110100 & -7.49537200 \\
\hline $\mathrm{H}$ & -2.14690100 & -2.02639600 & -5.87675600 \\
\hline $\mathrm{H}$ & -1.80904100 & -3.25175700 & -7.10895600 \\
\hline $\mathrm{H}$ & -1.76963800 & -3.68391000 & -5.38742200 \\
\hline $\mathrm{H}$ & 0.02634100 & -1.76474600 & -8.10161900 \\
\hline $\mathrm{H}$ & -0.29430900 & -0.52817500 & -6.87940900 \\
\hline $\mathrm{H}$ & 1.34511000 & -1.16903400 & -7.07528300 \\
\hline
\end{tabular}

(f) $\mathrm{Zn}^{+}(t \mathrm{BP}) \mathrm{TFSI}^{-}$ 


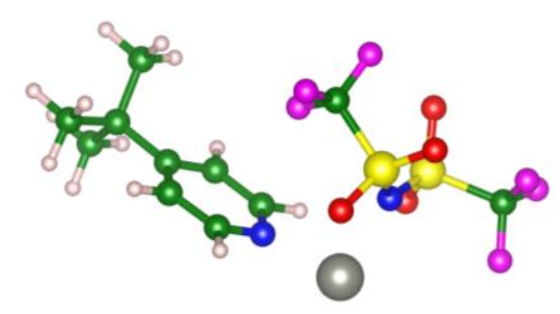

$\begin{array}{crrr}\text { Zn } & 0.51585200 & -2.40801100 & -1.29114300 \\ \mathrm{~N} & -0.86363500 & -2.55917600 & 0.32874900 \\ \mathrm{~S} & -1.70867900 & -3.89771600 & 0.76768100 \\ \mathrm{~S} & -0.89053900 & -1.09449400 & 1.07081000 \\ \mathrm{O} & -2.05377400 & -3.91549800 & 2.18612000 \\ \mathrm{O} & -2.20742100 & -0.53866800 & 1.34639300 \\ \mathrm{O} & -1.01392700 & -5.01814300 & 0.12598800 \\ \mathrm{O} & 0.11417300 & -0.34625600 & 0.28557700 \\ \mathrm{C} & -3.32964400 & -3.73196000 & -0.17591200 \\ \mathrm{C} & -0.07262800 & -1.33185600 & 2.77001900 \\ \mathrm{~F} & -3.07509400 & -3.55377300 & -1.47984400 \\ \mathrm{~F} & -4.03025400 & -2.69446400 & 0.28028500 \\ \mathrm{~F} & -4.03882600 & -4.85482800 & -0.01617700 \\ \mathrm{~F} & -0.96653200 & -1.67304800 & 3.68977700 \\ \mathrm{~F} & 0.49576300 & -0.16836300 & 3.11492900 \\ \mathrm{~F} & 0.88252100 & -2.27326200 & 2.70401800 \\ \mathrm{C} & 3.11200900 & -2.02067200 & 0.24816800 \\ \mathrm{C} & 2.19978200 & -4.12638400 & 0.52866400 \\ \mathrm{C} & 4.10303500 & -2.28120300 & 1.18892700 \\ \mathrm{H} & 3.04799700 & -1.05701800 & -0.24766700 \\ \mathrm{C} & 3.16132900 & -4.45450300 & 1.47163300 \\ \mathrm{H} & 1.40535500 & -4.81777400 & 0.26460700 \\ \mathrm{H} & 4.82025400 & -1.50204300 & 1.41165200 \\ \mathrm{H} & 3.11977700 & -5.43519400 & 1.93115100 \\ \mathrm{~N} & 2.17465700 & -2.92331900 & -0.07685500 \\ \mathrm{C} & 4.14739600 & -3.52247900 & 1.83719600 \\ \mathrm{C} & 5.18329900 & -3.87257400 & 2.90633400 \\ \mathrm{H} & 4.44242100 & -4.16564300 & 4.23201100 \\ \mathrm{H} & 5.96673400 & -5.12801000 & 2.45978100 \\ \mathrm{C} & 6.18253600 & -2.72809600 & 3.14372100 \\ \mathrm{H} & 3.86826800 & -3.29362300 & 4.56066900 \\ \mathrm{H} & 3.75098700 & -5.00736900 & 4.13455000 \\ \mathrm{H} & 5.16687800 & -4.41439500 & 5.01436000 \\ \mathrm{H} & 6.49135500 & -4.94784400 & 1.51582200 \\ \mathrm{H} & & -5.39010500 & 3.21940700\end{array}$




$\begin{array}{llll}\mathrm{H} & 5.31031800 & -5.99206800 & 2.32339800 \\ \mathrm{H} & 6.90261500 & -3.02838500 & 3.91062800 \\ \mathrm{H} & 6.74719800 & -2.48572400 & 2.23725900 \\ \mathrm{H} & 5.68478900 & -1.81940000 & 3.49759700\end{array}$

(g) $\mathrm{Zn}^{+} \mathrm{TFSI}^{-}$

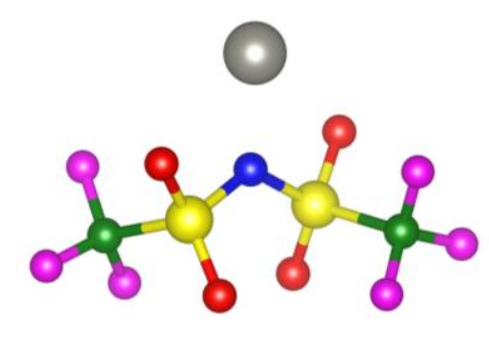

$\begin{array}{cccc}\text { Zn } & 0.392894 & -1.492715 & -3.526572 \\ \mathrm{~N} & 0.117685 & -0.824022 & -1.620946 \\ \mathrm{~S} & 1.549966 & -1.208401 & -0.890291 \\ \mathrm{O} & 1.467840 & -1.803912 & 0.431300 \\ \mathrm{O} & 2.242918 & -1.887851 & -2.017599 \\ \mathrm{C} & 2.462130 & 0.435945 & -0.708216 \\ \mathrm{~F} & 3.731730 & 0.171468 & -0.396139 \\ \mathrm{~F} & 2.416756 & 1.091600 & -1.875320 \\ \mathrm{~F} & 1.906358 & 1.176208 & 0.242404 \\ \mathrm{~S} & -1.263750 & -0.233148 & -0.894420 \\ \mathrm{O} & -0.962024 & 0.530305 & 0.307213 \\ \mathrm{O} & -2.076039 & 0.281030 & -1.991716 \\ \mathrm{C} & -2.126854 & -1.815795 & -0.340669 \\ \mathrm{~F} & -1.434998 & -2.417358 & 0.624075 \\ \mathrm{~F} & -2.234589 & -2.648853 & -1.389876 \\ \mathrm{~F} & -3.345684 & -1.505813 & 0.102393\end{array}$

(h) $\mathrm{Zn}^{2+}\left(\mathrm{TFSI}^{-}\right)_{2}$

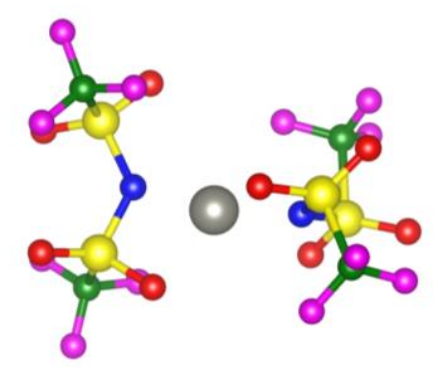

$\begin{array}{rrrr}\mathrm{Zn} & 0.36702200 & -1.67812400 & 0.43944000 \\ \mathrm{~N} & 2.25712400 & -1.94531200 & 0.08603500 \\ \mathrm{~N} & -1.46402400 & -1.37920800 & -0.13990100 \\ \mathrm{~S} & 3.08415900 & -2.65501100 & -1.19300500\end{array}$




$\begin{array}{lrrr}\text { S } & 2.86578200 & -0.93585400 & 1.24768700 \\ \text { O } & 2.22096900 & -3.73547000 & -1.64874200 \\ \text { O } & 4.49065800 & -2.81867600 & -0.86292500 \\ \text { O } & 1.55840800 & -0.47054200 & 1.82475200 \\ \text { O } & 3.89861400 & 0.00269100 & 0.85877900 \\ \text { C } & 3.58482900 & -2.07613100 & 2.57177600 \\ \text { C } & 2.94963500 & -1.30669900 & -2.50562500 \\ \text { F } & 3.43191400 & -1.77816000 & -3.65374900 \\ \text { F } & 3.63653000 & -0.22958100 & -2.13031400 \\ \text { F } & 1.65924800 & -0.98101000 & -2.65561700 \\ \text { F } & 3.83357800 & -1.34128600 & 3.65401000 \\ \text { F } & 4.70274800 & -2.63272200 & 2.12648300 \\ \text { F } & 2.68623300 & -3.01993900 & 2.85973400 \\ \text { S } & -2.19369500 & -2.55094500 & 0.77394800 \\ \text { S } & -2.20446700 & -0.18812000 & -1.06234300 \\ \text { O } & -3.39318300 & -2.19523400 & 1.50437000 \\ \text { O } & -3.53052800 & -0.61581500 & -1.47824700 \\ \text { O } & -0.96471100 & -3.05439300 & 1.47898800 \\ \text { O } & -1.17393700 & 0.28296500 & -1.97619300 \\ \text { C } & -2.63282300 & -3.93545600 & -0.43703800 \\ \text { C } & -2.41956900 & 1.18094700 & 0.21632200 \\ \text { F } & -1.57647300 & -4.16896200 & -1.21764300 \\ \text { F } & -3.67518800 & -3.57787300 & -1.17338500 \\ \text { F } & -2.92157100 & -5.02283000 & 0.27519700 \\ \text { F } & -3.26990000 & 0.79529000 & 1.16551700 \\ \text { F } & -2.88229500 & 2.26940000 & -0.39550300 \\ \text { F } & -1.22789600 & 1.44647600 & 0.76975600 \\ & & & \end{array}$

Figure $\mathbf{S} 7$ Figures and xyz coordinates of the optimized species. (a) spiro-MeOTAD, (b) spiro$\mathrm{MeOTAD}^{+} \mathrm{TFSI}^{-}$, (c) $\mathrm{Zn}^{+}(t \mathrm{BP})_{4}$, (d) $\mathrm{Zn}^{+}(t \mathrm{BP})_{3} \mathrm{TFSI}^{-}$, (e) $\mathrm{Zn}^{+}\left(t \mathrm{BP}_{2} \mathrm{TFSI}^{-}\right.$, (f) $\mathrm{Zn}^{+}(t \mathrm{BP}) \mathrm{TFSI}^{-}$, (g) $\mathrm{Zn}^{+} \mathrm{TFSI}^{-}$, and (h) $\mathrm{Zn}^{2+}\left(\mathrm{TFSI}^{-}\right)_{2}$. 


\section{Experimental}

Materials. All chemicals were purchased from Sigma-Aldrich, Merck and Acros, and used without further purification.

Preparation of spiro-MeOTAD solution. $70 \mathrm{mM}$ spiro-MeOTAD (99\%, Merck) solution in chlorobenzene was doped with the stock solutions $(1.7 \mathrm{M})$ of bis(trifluoromethylsulfonyl)imide lithium salt (LiTFSI) (99.95\%, Sigma-Aldrich) or bis(trifluoro-methylsulfonyl)imide zinc salt $\left(\mathrm{Zn}(\mathrm{TFSI})_{2}\right)(95 \%, \mathrm{TCI})$ in acetonitrile. Prior to dopant addition, spiro-MeOTAD was dissolved in chlorobenzene and $t \mathrm{BP}$ is added. For both of the dopants the molar concentration of the $\mathrm{TFSI}^{-}$was kept same. The molar ratio of additives for spiro-MeOTAD was 0.5 and 3.3 for $\mathrm{TFSI}^{-}$and $t \mathrm{BP}$, respectively.

Preparation of spiro-MeOTAD film. $\quad 30 \mu \mathrm{L}$ of spiro-MeOTAD solutions were deposited on quartz films by spin coating technique $(30 \mathrm{~s}, 1000 \mathrm{rpm}$ with an acceleration rate of $150 \mathrm{rpm}$ $\left.\mathrm{s}^{-1}\right)$.

Preparation of zinc complex. In order to see possible coordination among $\mathrm{Zn}(\mathrm{TFSI})_{2}$ (or LiTFSI) and $t \mathrm{BP}, \mathrm{Zn}(\mathrm{TFSI})_{2}$ (or LiTFSI) solution $(1.8 \mathrm{M}, 1 \mathrm{ml})$ in acetonitrile was stirred with excess $t \mathrm{BP}(0.46 \mathrm{~g}, 0.5 \mathrm{ml})$, at room temperature for 5 hours. The obtained viscous solution was dried in ambient air, and the resulting complex was collected as white solid. Zinc complex (50 mg) and $t$ BP $(20 \mu \mathrm{L})$ was dissolved in DMSO-d $6(0.5 \mathrm{~mL})$, respectively, for NMR measurements.

Measurements. Absorption spectroscopy measurements were performed with UV-Vis-NIR Spectrophotometer (Cary5, Varian). The film thickness was measured by using a profilometer (DektakXT Stylus Profiler, Bruker). FT-IR was measured by a Nicolet 6700 (ThermoFisher Scientific). ${ }^{1} \mathrm{H}$ NMR spectra were performed on Bruker AvanceIII-400 MHz NMR spectr ometer.

Theoretical calculation. DFT calculations of minimum-energy structure and molecular harmonic frequencies have been performed for all the species involved in Scheme 1, i.e. $\mathrm{Zn}^{2+}\left(\mathrm{TFSI}^{-}\right)_{2}$, spiro-MeOTAD, spiro-MeOTAD ${ }^{+} \mathrm{TFSI}^{-}, t \mathrm{BP}, \mathrm{TFSI}^{-}$, and $\mathrm{Zn}^{+}(t \mathrm{BP})_{\mathrm{m}}\left(\mathrm{TFSI}^{-}\right)_{\mathrm{n}}$ (with $\mathrm{n}=1$ and $\mathrm{m}=0,1,2,3$; or $\mathrm{n}=0$ and $\mathrm{m}=4$ ). We applied the B3LYP level of theory ${ }^{\mathrm{R} 1}$ with the SDD ECP and basis set for $\mathrm{Zn},{ }^{\mathrm{R} 2}$ and $6-31 \mathrm{G}(\mathrm{d})$ for the other atom species. ${ }^{\mathrm{R} 3}$ Dispersion interactions have been taken into account according to the DFT-D3 approach by Grimme, with the Becke-Johnson damping scheme. ${ }^{\mathrm{R} 4}$ The same level of theory has been successfully applied to study the electronic properties of Zn complexes. ${ }^{\mathrm{R} 5}$ All the calculations reported in this work were performed with the Gaussian 16 suite of programs for quantum chemistry. ${ }^{\text {R6 }}$ The detailed 
information of figures and xyz coordinates of the optimized species is shown in Figure S7. 


\section{References}

(R1) Becke, A. D. Density-Functional Thermochemistry. III. The Role of Exact Exchange. $J$. Chem. Phys. 1993, 98, 5648-5652.

(R2) Andrae, D.; Haeussermann, U.; Dolg, M.; Stoll, H.; Preuss, H. Energy-Adjusted ab initio Pseudopotentials for the Second and Third Row Transition-Elements. Theor. Chem. Acc. 1990, $77,123-141$.

(R3) Rassolov, V. A.; Ratner, M. A.; Pople, J. A.; Redfern, P. C.; Curtiss, L. A. 6-31G* Basis Set for Third-Row Atoms. J. Comp. Chem. 2001, 22, 976-984.

(R4) Grimme, S.; Ehrlich, S.; Goerigk, L. Effect of the Damping Function in Dispersion Corrected Density Functional Theory. J. Comp. Chem. 2011, 32, 1456-1465.

(R5) Minei, P.; Fanizza, E.; Rodríguez, A.M.; Muñoz-García, A.B.; Cimino, P.; Pavone, M.; Pucci A. Cost-Effective Solar Concentrators Based on Red Fluorescent Zn(II)Salicylaldiminato Complex. RSC adv. 2016, 6, 17474-17482.

(R6) Frisch, M. J.; Trucks, G. W.; Schlegel, H. B.; Scuseria, G. E.; Robb, M. A.; Cheeseman, J. R.; Scalmani, G.; Barone, V.; Petersson, G. A.; Nakatsuji, H. et al. Gaussian 16, Revision A.01, Gaussian, Inc., Wallingford CT, 2016. 